\title{
Resultados de un programa multidisciplinario de tratamiento intensificado de la Diabetes Mellitus tipo 1 (DM 1) en un Hospital Público
}

\author{
Ethel Codner D, Verónica Mericq G, Hernán G arcía B, \\ Claudia López $\mathbf{G}$, Jeannette Cáceres $V^{a}$, Ximena $\mathbf{G}$ aete $\mathbf{V}$, \\ Alejandra Avila $\mathrm{A}^{\mathrm{b}}$.
}

\section{Results of a multidisciplinary and intensified treatment program for type 1 Diabetes Mellitus in a Chilean Public Hospital}

Background: During the last decade, the importance of glycemic control in the prevention of the microvascular complications of type 1 Diabetes Mellitus (DM1) was clearly demonstrated. Aim: To evaluate the metabolic and anthropometric results of a multidisciplinary intensified treatment program of DMI in children and adolescents. Patients and methods: Report of 54 patients treated during 2001. The intensified treatment consisted of: multiple daily doses of insulin, frequent glycemic control, nutritional, psychological and educational support, and permanent availability of a diabetes nurse for telephonic support. Results: Thirty one patients were female, their mean age was $10.4 \pm 0.5$ years old and $52 \%$ were experiencing puberty. Fifty three percent of the patients used 3 insulin doses per day, 95\% changed rapid insulin dose based on glucose levels and 18\% considered carbohydrates in their rapid insulin dosing. Mean glycosilated hemoglobin was $8.18 \pm 0.23 \%$ without differences by sex or pubertal status. Sex, pubertal stage and the number of insulin doses did not contribute to glycosilated hemoglobin changes. There were no differences in weight or BMI, but there was a decrease in height $Z$ score from the admission to the program until the last control $(0.1 \pm 0.1$ vs $0.3 \pm 0.1 \mathrm{DS} ; \mathrm{p}<0.01$ ). Conclusions: A modified intensified modality of DM1 therapy for pediatric patients in a public hospital in Chile is feasible, achieving similar metabolic control, compared to international large centers (Rev Méd Chile 2003; 131: 857-64).

(Key Words: Diabetes Mellitus, insulin dependent; Health care surveys; Insulin)

\footnotetext{
Recibido el 13 de enero, 2003. Aceptado en versión corregida el 26 de junio, 2003. Instituto de Investigaciones Matemo Infantil (IDIMI), Facultad de Medicina, Universidad de Chile y Hospital Clínico San Borja Amiarán, Servicio de Salud Santiago Centro, Santiago, Chile. a Nutricionista clínica

benfermera Universitaria
}

Correspondencia a: Dra. Ethel Codner D. IDIMI, Universidad de Chile. Casilla 226-3, Santiago, Chile. Teléfono: 562-424-8280. Fax: 562-424-7240. E mail: ecodner@machi.med.uchile.cl 
$\mathrm{E}^{\mathrm{n}}$ los pacientes con DM1 el inicio del tratamiento con insulina, en 1922, logró disminuir la mortalidad por complicaciones agudas y aumentó su sobrevida, pero hizo evidente la aparición, a largo plazo, de complicaciones microvasculares ${ }^{1}$. El Diabetes Control and Complications Trial (DCCT) en DM1 y el United Kingdom Prospetive Diabetes Study (UKPDS) en DM2 demostraron claramente la importancia que tienen los niveles de glicemia en la aparición y progresión de las complicaciones microvasculares en adolescentes y adultos ${ }^{2,3}$. En el DCCT los pacientes fueron tratados con múltiples dosis 0 bomba de infusión de insulina, y además se incorporó un equipo multidisciplinario consistente en médicos, enfermeras, nutricionistas, asistentes sociales y psicólogos que brindaron el apoyo óptimo para lograr niveles de glicemias normales. Por otra parte, este estudio produjo un aumento en las exigencias del tratamiento de la enfermedad, obligando a los pacientes a realizar múltiples inyecciones de insulina y controles de glicemia.

En el DCCT se observó que los adolescentes entre los 13 y 17 años, a pesar de las mayores dificultades que tienen en lograr un control glicémico óptimo, presentaron los mismos beneficios que los adultos en cuanto a la prevención de las complicaciones microvasculares ${ }^{4}$, y que éstos perduraron en el tiempo ${ }^{5,6}$. En los menores de 13 años no existe un estudio semejante que permita extender las conclusiones del DCCT. Más aún, los pacientes menores de 7 años presentan con mayor frecuencia hipoglicemia y alteraciones neuro-cognitivas, lo que ha motivado que los niveles deseables de glicemia sean más flexibles e individualizados ${ }^{7-10}$.

El tratamiento intensificado requiere un equipo multidisciplinario capaz de motivar y educar a los pacientes en un tratamiento de múltiples dosis de insulina, control glicémico frecuente y entrenamiento nutricional ${ }^{11}$. El año 1999 iniciamos un programa multidisciplinario de intensificación del tratamiento de la DM1 en niños y adolescentes que se encontraban en control en el Hospital Clínico San Borja-Arriarán. El objetivo de este trabajo es comunicar los resultados obtenidos en cuanto a control metabólico y antropometría en nuestro grupo de pacientes infanto-juveniles con DM1 después de dos años de iniciado dicho programa.

\section{PACIENTES y MÉTOdo}

Diseño del estudio. Se muestran los resultados del grupo total de pacientes con DM1 menores de 18 años en control durante el año 2001 en el Instituto de Investigaciones Materno Infantil (IDIMI), localizado en el Hospital San Borja Arriarán (n:54) tratados con la modalidad terapéutica que se describe a continuación.

Modalidad de tratamiento intensificado

Insulinoterapia y autocontrol de glicemia. Se modificó el esquema de tratamiento con insulina a un esquema de multidosis con, al menos, tres inyecciones diarias de insulina. La mayoría fue tratado con una dosis matinal y vespertina de mezcla de insulina intermedia (NPH) y cristalina, y una tercera inyección de insulina cristalina. En algunos pacientes se requirió el uso de 3 dosis diarias de $\mathrm{NPH}$. Los criterios para aumentar el número de inyecciones al día fueron los siguientes: Hemoglubina Glicosilada A1C (HbA1C) mayor de $8 \%$, hiperglicemia durante la tarde, u horarios de comida y actividad muy variables. El aumento del número de inyecciones diarias se realizó preferentemente en los pacientes mayores de 7 años, y se reservó sólo para casos muy seleccionados en pacientes menores a esta edad. Se adiestró a los pacientes a cambiar la dosis de insulina cristalina según la glicemia, y a considerar la ingesta de hidratos de carbonos, junto con la glicemia, para decidir la dosis de insulina cristalina. Todos los pacientes o sus padres fueron entrenados en el aporte de dosis adicionales de insulina en caso de necesidad ( «efuerzos adicionales»). Todos los pacientes realizaron medición de glicemia en forma ambulatoria al menos 3 ó 4 veces al día, con lectura automática en máquina Accutrend Sensor. Además se entrenó a los pacientes o sus familias para manejar la insulinoterapia durante los días con enfermedades intercurrentes.

Tratamiento nutricional. Se realizó entrenamiento nutricional según las recomendaciones de la American Diabetes Association del año 1994², con varias sesiones individuales consecutivas hasta que la madre o el paciente reconocieran la cantidad de hidratos de carbono de los principales alimentos y fueran capaces de establecer porcio- 
nes de intercambio. El objetivo de esta educación fue mantener constante las porciones de hidrato de carbono, con el fin de conseguir la mayor estabilidad posible en el aporte, y el reconocimiento de los cambios de ingesta para adecuar la dosis de cristalina. Junto con el entrenamiento individual, se realizaron actividades grupales de educación en nutrición en forma permanente.

Apoyo de enfermería y control médico. Una enfermera diplomada en diabetes (AA) brindó apoyo telefónico permanente por medio de teléfono celular, $24 \mathrm{~h}$ del día, para cambio de dosis y manejo de urgencias. Dicha enfermera coordinó las siguientes actividades: sesiones educativas mensuales a los padres, talleres para los pacientes según su edad, entrenamiento de madres monitoras en educación, entrenamiento de adolescentes monitores, actividades recreativas anuales para todo el grupo de enfermos, y sesiones educativas personales si fuera necesario. Existió disponibilidad de control médico frecuente según necesidad de los pacientes para ambos grupos.

Apoyo de salud mental y asistencia social. Alumnos en práctica de sicología realizaron terapia de grupo e individual, junto con el apoyo de la asistente social del hospital.

Control metabólico y de crecimiento. Se evaluó la hemoglobina glicosilada A1C con el método Bayer DCA-2000 en forma trimestral. Se analizó el control metabólico logrado según el promedio de la $\mathrm{HbA1C}$ de todos los exámenes efectuados durante el año 2001. Se estudió la repercusión sobre el crecimiento evaluando el último control clínico del año 2001, usando los índices talla/edad e índice de masa corporal (IMC) según las curvas NCHS 200013, expresadas en desviaciones estándares (DS) para la edad y sexo (Score Z). Se clasificó el estadio puberal según los criterios de Marshall y Tanner, y se consideraron los sujetos como prepuberales cuando el volumen testicular era menor de $4 \mathrm{cc}$ en los varones o las mamas se encontraban en estadio de Tanner I en las mujeres ${ }^{14,15}$.

Evaluación de los resultados y análisis estadístico. El análisis estadístico se efectuó con prueba de T no pareada o Mann Whitney, para los datos paramétricos y no paramétricos, respectivamente, o estudio de proporciones con prueba exacta de Fisher. Se utilizó el análisis de covarianza con un modelo lineal general para evaluar el efecto de los diferentes parámetros sobre la HbA1C. Se consideró significativo un valor $p \leq 0,05$. Los resultados se muestran como promedio \pm error estándar.

\section{RESULTADOS}

El grupo de pacientes con DM1 controlados en el IDIMI con la modalidad intensificada constó de 54 niños y adolescentes, con una edad promedio de 10,45 $\pm 0,5$ años (31 mujeres) y una duración de la diabetes de 3,69 $\pm 0,41$ años. Veintiocho de ellos eran puberales $(51,9 \%)$ (Tabla 1$)$. Veintinueve $(53,7 \%)$ pacientes requirieron tratamiento con tres dosis diarias de insulina, 23 pacientes fueron tratados con dos dosis diarias y un paciente estaba manejado con una dosis diaria. De los pacientes tratados con tres dosis diarias, $72,4 \%$ eran púberes, $79,3 \%$ eran capaces de intercambiar hidratos de carbono, 100\% modificó la dosis de insulina cristalina según la glicemia, aunque sólo 13,8\% consideró la ingestión de hidratos de carbono en su indicación de insulina cristalina. Se usó un esquema de dos inyecciones de mezcla de NPH más cristalina y una tercera inyección de insulina cristalina en 24 pacientes, y de tres inyecciones de mezcla de NPH y cristalina en cuatro sujetos. En el grupo tratado con dos dosis diarias, 58,3\% realizaba correctamente el intercambio de hidratos de carbono, 91,7\% modificaban la dosis de insulina según glicemia, y 20,8\% consideraba la ingesta de hidratos de carbono en su decisión de la dosis de insulina. Este último grupo de pacientes fue tratado con dos dosis diarias de mezcla de insulina NPH y cristalina.

El grupo total de pacientes presentó una HbA1C de $8,18 \pm 0,23 \%$, y requirió un aporte diario de insulina de $0,8 \mathrm{U} / \mathrm{kg} /$ día. El nivel de HbA1C logrado en los pacientes con tres dosis fue $8,05 \pm 0,33 \%$, y en los con dos dosis fue $8,34 \pm 0,32 \%(\mathrm{p}=0,052)$. El control metabólico obtenido en los diferentes grupos con 2 y 3 dosis se detalla en la Tabla 2. La HbA1C en prepúberes fue $8,11 \pm 0,32 \%$ y en los púberes fue $8,25 \pm 0,34(p=0,76)$. No existieron diferencias significativas al comparar varones con mujeres. Los pacientes púberes comparados con los prepúberes requirieron 
Tabla 1. C aracterísticas de los pacientes estudiados y del tratamiento efectuado

\begin{tabular}{|lc|}
\hline & Intensificado (n: 54) \\
\hline Edad (años) & $10,45 \pm 0,50$ \\
Tiempo de duración de la DM1 (años) & $3,69 \pm 0,41$ \\
Varones (\%) & $23(42,6)$ \\
$\quad$ Púberes (\%) & $10(43)$ \\
Mujeres (\%) & $31(57,4 \%)$ \\
$\quad$ Púberes (\%) & $18(58,1)$ \\
Pacientes con 3 dosis (\%) & $29(53,7)$ \\
$\quad$ Púberes (\%) & $21(72,4)$ \\
$\quad$ Intercambia hidratos de carbono (\%) & $23(79,3)$ \\
$\quad$ Cambia dosis de cristalina según glicemia (\%) & $29(100)$ \\
$\quad$ Cambia dosis de cristalina según ingesta de hidratos de carbono (\%) & $4(13,8)$ \\
$\quad$ Usa dos dosis diarias de NPH y tres de cristalina (\%) & $22(75,9)$ \\
$\quad$ Usa tres dosis diarias de NPH y cristalina (\%) & $4(13,8)$ \\
Pacientes con dos dosis al día & $24(46,3)$ \\
$\quad$ Púberes (\%) & $7(29,2)$ \\
$\quad$ Intercambia hidratos de carbono (\%) & $14(58,3)$ \\
$\quad$ Cambia dosis de cristalina según glicemia (\%) & $22(91,7)$ \\
$\quad$ Cambia dosis de cristalina según ingesta de hidratos de carbono (\%) & $5(20,8)$ \\
$\quad$ Usa dos dosis de NPH y cristalina (\%) & $24(100)$ \\
Pacientes con una dosis al día (\%) & $1(1,9)$ \\
\hline
\end{tabular}

Tabla 2. C aracterísticas del tratamiento efectuado y control metabólico logrado en los niños y adolescentes en control en el H ospital San Borja Arriarán después de dos años de iniciar un programa de intensificación del tratamiento

\begin{tabular}{|lcccccc|}
\hline & $\mathrm{N}$ & HbA1C & Edad (años) & № Dosis al día & $\begin{array}{c}\text { Aporte de } \\
\text { insulina día } \\
(\mathrm{U} / \mathrm{kg})\end{array}$ & $\begin{array}{c}\text { Pacientes }>2 \\
\text { dosis/día (\%) }\end{array}$ \\
\hline Grupo total de pacientes & 54 & $8,18 \pm 0,23$ & $10,45 \pm 0,50$ & $2,51 \pm 0,07$ & $0,80 \pm 0,04$ & $28(51,9)$ \\
Varones & 23 & $8,31 \pm 0,28$ & $10,28 \pm 0,85$ & $2,39 \pm 0,13$ & $0,77 \pm 0,04$ & $10(43,5)$ \\
Mujeres & 31 & $8,09 \pm 0,34$ & $10,58 \pm 0,62$ & $2,6 \pm 0,09$ & $0,83 \pm 0,06$ & $18(58,06)$ \\
Prepúberes & 26 & $8,11 \pm 0,32$ & $7,4 \pm 0,44$ & $2,27 \pm 0,1 *$ & $0,68 \pm 0,05 * *$ & $8(30,8) * * * *$ \\
Púberes & 28 & $8,25 \pm 0,34$ & $13,29 \pm 0,42$ & $2,74 \pm 0,86$ & $0,92 \pm 0,05$ & $20(71,42)$ \\
Pacientes con $\leq 2$ dosis & 25 & $8,34 \pm 0,32$ & $8,9 \pm 0,82$ & $1,96 \pm 0,04$ & $0,71 \pm 0,04$ & $0(0)$ \\
Pacientes con 3 dosis & 29 & $8,05 \pm 0,33$ & $11,79 \pm 0,5$ & $3,0 \pm 0,0$ & $0,87 \pm 0,06$ & $29(100)$ \\
\hline
\end{tabular}

*: $p<0,001$ prepúberes vs púberes. **. $p<0,01$ prepúberes vs púberes. ***: $p<0,05$ prepúberes vs púberes.

mayor número de dosis al día $(2,74+0,86$ vs $2,27 \pm 0,1$ inyecciones/día, $\mathrm{p}=0,0007)$ y mayor dosis por kilo de insulina $(0,92 \pm 0,05$ vs $0,68 \pm 0,05 \mathrm{U} / \mathrm{kg} /$ día, $\mathrm{p}=0,0011)$. El grupo puberal efectuó tratamiento con multidosis en una proporción mayor que los prepúberes $(71,42$ vs $30,8 \%, p=0,011$ ).
Se realizó un análisis de covarianza para ver el efecto de las diferentes variables sobre la hemoglobina glicosilada. El sexo, el estadio puberal y el número de dosis no contribuyeron significativamente a la varianza de la hemoglobina glicosilada. 
Al comparar la antropometría de los pacientes en el último control efectuado con aquélla al momento del diagnóstico de la enfermedad, se observó una tendencia a un alza del IMC que no alcanzó significancia estadística. Existió una disminución significativa del Score Z de talla desde el debut de $0,10 \pm 0,14$ DS, a $-0,26 \pm 0,13$ DS en el último control del año $2001(p=0,0021)$. El grupo de pacientes púberes no presentó cambios en la antropometría con respecto al debut, sin embargo, los pacientes prepúberes presentaron una disminución del Score $\mathrm{Z}$ de la talla desde $0,22 \pm 0,21$ a $-0,27 \pm 0,22$ DS. Los resultados antropométricos se detallan en la Tabla 3.

\section{DisCUSIÓN}

En este estudio se muestran los resultados de un programa de intensificación del tratamiento de la DM1 de dos años de duración en niños y adolescentes en un hospital público chileno. Nuestros pacientes lograron una HbA1C promedio de 8,18\%, lo que es semejante a lo reportado para este grupo etario en otros centros del mundo (Tabla 4). El trabajo pionero de esta nueva forma de manejo de la diabetes fue el DCCT que logró una HbA1C de $7,3 \%$ después de un promedio de 6,5 años de

Tabla 3. Antropometría de los pacientes al debut y al último control del año 2001

\begin{tabular}{|lcc|}
\hline & Debut & 2001 \\
\hline Grupo total & & \\
Peso/edad (Score Z) & $0,39 \pm 0,13$ & $0,35 \pm 0,11$ \\
Talla/edad (Score Z) & $0,10 \pm 0,14$ & $-0,26 \pm 0,13^{*}$ \\
IMC (Score Z) & $0,45 \pm 0,14$ & $0,64 \pm 0,10$ \\
& & \\
Puberales & & \\
Peso/edad (Score Z) & $0,16 \pm 0,19$ & $0,27 \pm 0,13$ \\
Talla/edad (Score Z) & $-0,05 \pm 0,18$ & $-0,27 \pm 0,17$ \\
IMC (Score Z) & $0,24 \pm 0,25$ & $0,49 \pm 0,13$ \\
& & \\
Prepúberes & & \\
Peso/edad (Score Z) & $0,58 \pm 0,17$ & $0,44 \pm 0,18$ \\
Talla/edad (Score Z) & $0,22 \pm 0,21$ & $-0,27 \pm 0,22^{*}$ \\
IMC (Score Z) & $0,62 \pm 0,15$ & $0,84 \pm 0,16$ \\
\hline
\end{tabular}

IMC: índice de masa corporal.

${ }^{*} p<0,01$. seguimiento ${ }^{2}$. Sin embargo, al analizar por separado el grupo entre 13 y 17 años del DCCT, la HbA1C promedio fue $8,1 \%{ }^{4}$. En los últimos años los centros americanos reportan valores entre 7,8 y 8,8\%16,17. El estudio de Hvidore ha seguido el control metabólico en dos periodos (1995 y 1998) en 21 centros de Europa, Canadá y Japón y obtuvo una $\mathrm{HbA1C}$ promedio de $8,6 \% 18$, demostrando que los resultados de cada uno de los centros se correlacionó con sus resultados 3 años después, sugiriendo que además del factor biológico, factores étnicos, culturales y del equipo tratante (afiatamiento del grupo, modelos educativos, apoyo, disponibilidad, coordinación) parecen ser importantes en los resultados obtenidos ${ }^{18,19}$.

Durante la adolescencia es frecuente que el control glicémico se deteriore debido a la insulinorresistencia fisiológica de esta etapa ${ }^{20,21}$ y a factores psicológicos que disminuyen la adherencia a la terapia. Sin embargo, la pubertad es el momento en que el adecuado control metabólico adquiere aún mayor relevancia, ya que éste es el período de aparición de complicaciones cróni$\mathrm{cas}^{22}$. En nuestra serie es destacable el hecho que el control metabólico que presentaron los sujetos puberales fue similar al grupo prepuberal, lo que se explicaría por el mayor número de dosis y dosis/kg de insulina utilizadas por este grupo, como a su vez por el mejor manejo de la flexibilidad de dosis. En el grupo prepuberal, a pesar de utilizar con mayor frecuencia un esquema de sólo 2 dosis de insulina (Tabla 2), probablemente la educación en la flexibilidad de dosis de acuerdo a variables como glicemia e ingesta de HC, permitió obtener resultados comparables a las series extranjeras. En este grupo fuimos especialmente cuidadosos con la utilización de los regíme-

Tabla 4. Niveles de H bA1C logrados en adolescentes en diferentes centros del mundo

\begin{tabular}{|lc|}
\hline & HbA1C (\%) \\
\hline Chile, IDIMI & 8,18 \\
DCCT adolescentes (4) & 8,1 \\
USA, 25 centros (16) & $7,8-8,8$ \\
Grey et al, Universidad de Yale (17) & 7,9 \\
Grupo internacional Hvidore (18) & 8,6 \\
\hline
\end{tabular}


nes de insulina, por la dificultad que se presenta para mantener la casi euglicemia sin presentar hipoglicemia. Al analizar nuestros datos por sexo, no encontramos diferencias en el control metabólico, aunque existen reportes de un posible dimorfismo sexual en el metabolismo glucídico ${ }^{23}$.

El crecimiento, así como la maduración sexual, de los pacientes diabéticos pueden verse comprometidos si el tratamiento no es óptimo. Con la mejoría del control metabólico, el deterioro severo del crecimiento se ha convertido en una excepción, pero aún se observan alteraciones en la composición corporal ${ }^{24}$ y en el crecimiento estatu$\mathrm{ral}^{25-27}$. Existen estudios que demuestran que puede existir pérdida de talla durante la niñez ${ }^{28}$, pero al parecer ésta se produciría fundamentalmente durante el estirón puberal ${ }^{29,30}$. En el grupo de pacientes que aquí reportamos la estatura en el grupo puberal expresada en SDS no mostró cambios respecto al debut, sin embargo hubo un deterioro leve pero significativo en el grupo prepuberal. Sólo el seguimiento a largo plazo de estos pacientes, nos permitirá evaluar el efecto de la terapia intensificada sobre el crecimiento lineal ${ }^{28-30}$

Uno de los inconvenientes que se ha observado en el uso de la terapia intensificada es el incremento de peso e IMC de los pacientes. Se ha planteado que este aumento del peso podría tener efectos negativos sobre la mortalidad cardiovascular, ya que generalmente se acompaña de alteraciones en el perfil lipídico ${ }^{31}$. Sin embargo, el efecto de la optimización de las glicemias sobre la mortalidad cardiovascular aún es controvertido 2,32,33. En este estudio el peso (SDS) de los pacientes después de 2 años de terapia intensificada no mostró diferencias comparado al de aquél después de la recuperación del debut, sin embargo hubo una tendencia a un mayor IMC que no alcanzó significancia. Esto último concuerda con lo observado en otras series $^{4,31,34,35}$. Los otros dos mayores inconvenientes del tratamiento intensificado son el aumento de la incidencia de hipoglicemia ${ }^{2}$ y el incremento del costo del tratamiento ${ }^{36}$. En el DCCT los pacientes que presentaron hipoglicemia severa no tuvieron secuelas neurológicas ni electrocardiográficas ${ }^{2,37}$. Aunque es importante recalcar en este punto que los pacientes participantes en el DCCT eran todos mayores de 13 años. El período más sensible en el desarrollo neurológico coincide con los primeros 4 años de vida. Se desconoce aún el efecto que la intensificación de la terapia puede tener en la función cognitiva a largo plazo en este grupo etario, aunque recientemente existen evidencias que al menos a mediano plazo en niños mayores de 6 años no habría efectos deletéreos ${ }^{38-40}$.

El aumento de los costos en 2 ó 3 veces $^{36}$ es el otro gran problema al tratar de establecer esta forma de terapia. Sin embargo, creemos que la relación costo-beneficio a largo plazo lo hace altamente rentable para las instituciones de salud $36,41-44$. Se estima que si se trataran 120 pacientes con DM1 con tratamiento intensificado resultaría en una ganancia de 691 años libre de insuficiencia renal terminal, 678 años libres de amputación, y de 920 años sin ceguera ${ }^{36,41-44}$.

El manejo intensificado tiene por objetivo administrar una substitución de insulina lo más parecida a la fisiológica, en que se aporte una insulina 〈basal» y otra «prandial» antes de las comidas $^{11}$. Nuestra forma de realizar tratamiento intensificado con una mezcla de NPH e insulina cristalina tiene diversas limitaciones, y se aleja del esquema intensificado ideal ${ }^{45,46}$. Esto se refleja en que los pacientes pierden algo de libertad en los horarios de comidas con respecto a lo que pueden otorgar otras insulinas como los análogos lyspro o glargina, que actualmente no están disponibles en los servicios estatales de salud ${ }^{4}$.

Nuestro trabajo no evaluó el efecto de la intensificación del tratamiento sobre la calidad de vida, pero existen evidencias que los sujetos con mejor control metabólico presentan una mejoría en su calidad de vida y satisfacción con el tratamiento de la diabetes al disponer de una forma de terapia con mayor flexibilidad ${ }^{47}$.

En conclusión, mostramos que es posible instaurar un esquema de tratamiento intensificado modificado en población infanto-juvenil de un hospital público chileno, logrando control glicémico semejante a lo reportado en otros centros del mundo. Se observaron beneficios en los aspectos metabólicos. Esperamos que en el futuro logremos optimizar dicho tratamiento con otras formas de insulinoterapia, mayor cohesión del equipo profesional, y nuevas técnicas educativas que representen mejorías en la calidad de vida de nuestros pacientes. 


\section{REFERENCIAS}

1. Nathan DM. Long term complications of diabetes mellitus. N Engl J Med 1993; 328: 1676-85.

2. The Diabetes Control and Complcations Trial ReSEARCH GROUP. The effect of intensive treatment of diabetes on the development and progression of long term complications in insulin dependent diabetes mellitus. The Diabetes Control and Complications Trial Research Group. N Engl J Med 1993; 329: 977-86.

3. UK Prospective Diabetes Study (UKPDS) Group. Intensive blood glucose control with sulphonylureas or insulin compared with conventional treatment and risk of complications in patients with type 2 diabetes (UKPDS 33). Lancet 1998; 352: 837-53.

4. The Diabetes Control and Complications Trial ReSEARCH GROUP. Effect of intensive diabetes treatment on the development and progression of long term complications in adolescents with insulin dependent diabetes mellitus: Diabetes Control and Complications Trial. Diabetes Control and Complications Trial Research Group. J Pediatr 1994; 125: 177-88.

5. The Diabetes Control and Complations Trial ReSEARCH Group. Beneficial effects of intensive therapy of diabetes during adolescence: Outcomes after the conclusion of the Diabetes Control and Complications Trial (DCCT). J Pediatr 2001; 139: 804-12.

6. The Diabetes Control and Complications Trial ReSEARCH GROUP. Retinopathy and nephropathy in patients with type 1 diabetes four years after a trial of intensive therapy. The Diabetes Control and Complications Trial/Epidemiology of Diabetes Interventions and Complications Research Group. N Engl J Med 2000; 342: 381-9.

7. Mortensen HB, Hougaard P. Comparison of metabolic control in a cross sectional study of 2,873 children and adolescents with IDDM from 18 countries. The Hvidore Study Group on Childhood Diabetes. Diabetes Care 1997; 20: 714-20.

8. Ryan C, Vega A, Drash A. Cognitive deficits in adolescents who developed diabetes early in life. Pediatrics 1985; 75: 921-7.

9. Bjorgaas M, Gimse R, Vik T, SAND T. Cognitive function in type 1 diabetic children with and without episodes of severe hypoglycaemia. Acta Paediatr 1997; 86: 148-53.

10. American Diabetes Association. Standards of Medi- cal Care for Patients with Diabetes Mellitus. Diabetes Care 2002; 25: 33S-49.

11. HIRSCH IB. Intensive treatment of type 1 diabetes. Med Clin North Am 1998; 82: 689-719.

12. Franz MJ, Horton ES SR, Bantle JP, Beebe CA, BRunzell JD, Coulston AM et al. Nutrition principles for the management of diabetes and related complications. Diabetes Care 1994; 17: 490-518.

13. Ogden CL, Kuczmarski RJ, Fifgal KM, Mei Z, Guo S, WEI R ET AL. Centers for Disease Control and Prevention 2000 growth charts for the United States: improvements to the 1977 National Center for Health Statistics version. Pediatrics 2002; 109: 45-60.

14. Marshall WA, TANNER JM. Variations in pattern of pubertal changes in girls. Arch Dis Child 1969; 44: 291-303.

15. Marshall WA, TanNer JM. Variations in the pattern of pubertal changes in boys. Arch Dis Child 1970; 45: 13-23.

16. Rosenbloom AL, Schatz DA, Krischer JP, Skyler JS, BECKER DJ, LAPORTE RE ET AL Therapeutic controversy: prevention and treatment of diabetes in children. J Clin Endocrinol Metab 2000; 85: 494-522.

17. Grey M, Boland EA, Davidson M, Yu C, SumvanBolyai S, Tamborlane WV. Short term effects of coping skills training as adjunct to intensive therapy in adolescents. Diabetes Care 1998; 21: 902-8.

18. Danne T, Mortensen HB, Hougaard P, Lynggaard H, Aanstoot HJ, Chiarem F et al. Persistent differences among centers over 3 years in glycemic control and hypoglycemia in a study of 3,805 children and adolescents with type 1 diabetes from the Hvidore Study Group. Diabetes Care 2001; 24: 1342-7.

19. Ilonen J, Reijonen H, Knip M, Simell O. Population based genetic screening for IDDM susceptibility as a source of HLA-genotyped control subjects. Diabetologia 1996; 39: 123.

20. Morán A, Jacobs DR Jr, Steinberger J, Hong CP, PRINEAs R, LuePKER R ET aL. Insulin resistance during puberty: results from clamp studies in 357 children. Diabetes 1999; 48: 2039-44.

21. Amiel SA, Caprio S, Sherwin RS, Piewe G, Haymond MW, TAMBORLANE WV. Insulin resistance of puberty: a defect restricted to peripheral glucose metabolism. J Clin Endocrinol Metab 1991; 72: 277-82.

22. Murphy RP, Nanda M, Plotnick L, Enger C, Vitale S, Patz A. The relationship of puberty to diabetic retinopathy. Arch Ophthalmol 1990; 108: 215-8.

23. Hoffman RP, Vicini P, Sivitz WI, Coвeш C. Pubertal adolescent male-female differences in insulin 
sensitivity and glucose effectiveness determined by the one compartment minimal model. Pediatr Res 2000; 48: 384-8.

24. Dunger D, Ahmed L, Ong K. Growth and body composition in type 1 diabetes mellitus. Horm Res 2002; 58 Suppl 1: 66-71.

25. ConNors MH. Growth in the diabetic child. Pediatr Clin North Am 1997; 44: 301-6.

26. JACKSON RL Growth and maturation of children with insulin dependent diabetes mellitus. Pediatr Clin North Am 1984; 31: 545-67.

27. Brown DC, Stirling HF, Butler GE, Kelnar CJ, Wu FC. Differentiation of normal male prepuberty and hypogonadotrophic hypogonadism using an ultrasensitive luteinizing hormone assay. Horm Res 1996; 46: 83-7.

28. Brown M, Ahmed ML, Ciayton KL, Dunger DB. Growth during childhood and final height in type 1 diabetes. Diabet Med 1994; 11: 182-7.

29. Danne T, Kordonouri O, Enders I, Weber B. Factors influencing height and weight development in children with diabetes. Results of the Berlin Retinopathy Study. Diabetes Care 1997; 20: 281-5.

30. Sepúlveda A, PéreZ J, Melia I. Crecimiento en niños con diabetes mellitus insulino dependientes. Rev Chil Pediatr 1997; 68: 61-5.

31. Purnell JQ, Hokanson JE, Marcovina SM, Stefres MW, Cleary PA, Brunzell JD. Effect of excessive weight gain with intensive therapy of type 1 diabetes on lipid levels and blood pressure: results from the DCCT. Diabetes Control and Complications Trial. Jama 1998; 280: 140-6.

32. NatHAN DM. Clinical review 146: The impact of clinical trials on the treatment of diabetes mellitus. J Clin Endocrinol Metab 2002; 87: 1929-37.

33. The Diabetes Control and Complications Trial ReSEARCH GROUP. Effect of intensive diabetes management on macrovascular events and risk factors in the Diabetes Control and Complications Trial. Am J Cardiol 1995; 75: 894-903.

34. The Diabetes Control and Complications Trial ReSEARCH GROUP. Weight gain associated with intensive therapy in the diabetes control and complications trial. The DCCT Research Group. Diabetes Care 1988; 11: 567-73.

35. Greene AC, Tripaldi M, Chiareu F, McKiernan P, MorRis A, NEWTON R ET AL. Cross cultural differences in the management of children and adolescents with diabetes. Horm Res 2002; 57 Suppl 1: 75-7.

36. HeRman WH, Eastman RC. The effects of treatment on the direct costs of diabetes. Diabetes Care
1998; 21 Suppl 3: C19-24.

37. Kramer L, Fasching P, Madl C, Schneider B, Damjancic P, Waldhausl W et al. Previous episodes of hypoglycemic coma are not associated with permanent cognitive brain dysfunction in IDDM patients on intensive insulin treatment. Diabetes 1998; 47: 1909-14.

38. Wysocki T, Harris Ma, Mauras N, Fox L, Taylor A, JACKSON SC ET AL. Absence of adverse effects of severe hypoglycemia on cognitive function in school aged children with diabetes over 18 months. Diabetes Care 2003; 26: 1100-5.

39. Shah SC, Malone JI, Simpson NE. A randomized trial of intensive insulin therapy in newly diagnosed insulin dependent diabetes mellitus. N Engl J Med 1989; 320: 550-4.

40. The Diabetes Control and Complications Trial ReSEARCH GROUP. Effect of intensive therapy on residual beta cell function in patients with type 1 diabetes in the diabetes control and complications trial. A randomized, controlled trial. The Diabetes Control and Complications Trial Research Group. Ann Intern Med 1998; 128: 517-23.

41. Zinman B. Translating the Diabetes Control and Complications Trial (DCCT) into clinical practice: overcoming the barriers. Diabetologia 1997; 40 Suppl 2: S88-90.

42. The Diabetes Control and Complications Trial ReSEARCH GROUP. Lifetime benefits and costs of intensive therapy as practiced in the diabetes control and complications trial. The Diabetes Control and Complications Trial Research Group. Jama 1996; 276: 1409-15.

43. The Diabetes Control and Complications Trial ReSEARCH GROUP. Resource utilization and costs of care in the diabetes control and complications trial. Diabetes Care 1995; 18: 1468-78.

44. Dagogo-JACK S. DCCT results and diabetes care in developing countries. Diabetes Care 1995; 18: 416-7.

45. Ratner RE, Hirsch IB, Neifing JL, Garg SK, Mecca TE, WILSON CA. Less hypoglycemia with insulin glargine in intensive insulin therapy for type 1 diabetes. US Study Group of Insulin Glargine in Type 1 Diabetes. Diabetes Care 2000; 23: 639-43.

46. Schober E, Schoente E, Van Dyk J, Wernicke-Panten $\mathrm{K}$. Comparative trial between insulin glargine and $\mathrm{NPH}$ insulin in children and adolescents with type 1 diabetes. Diabetes Care 2001; 24: 2005-6.

47. MorIENSEN HB. Findings from the Hvidore Study Group on Childhood Diabetes: metabolic control and quality of life. Horm Res 2002; 57 Suppl 1: 117-20. 